\title{
A Contentious Trinity: Levels of Entailment in Brandom's Pragmatist Inferentialism
}

\author{
Edgar Andrade-Lotero • Catarina Dutilh Novaes
}

Received: 4 February 2010 / Revised: 20 July 2010 / Accepted: 21 January 2011 /

Published online: 11 February 2011

(C) The Author(s) 2011. This article is published with open access at Springerlink.com

\begin{abstract}
We investigate the relations among Brandom's three dimensions of semantic inferential articulation, namely, incompatibility entailments, committive consequences, and permissive consequences. In his unpublished manuscript "Conceptual Content and Discursive Practice" Brandom argues that (1) incompatibility entailment implies committive consequence, and that (2) committive consequence in turn implies permissive consequence. We criticize this hierarchy both on internal and external grounds. Firstly, we prove that, using Brandom's own definitions, the reverse of (1) also holds, and that the reverse of (2) may hold (but the proof relies on substantive assumptions). This suggests that there are no three different notions of inference emerging from Brandom's definitions, but at most two, and perhaps even just one. Secondly, this result puts into question the connections between the three inferential relations and the familiar notions of deduction and induction.
\end{abstract}

Keywords Incompatibility entailment - Committive consequence • Permissive consequence $\cdot$ Inferentialism

\footnotetext{
E. Andrade-Lotero $(\varangle)$

Faculty of Natural Sciences and Mathematics, Universidad Del Rosario, Bogota, D.C., Colombia

e-mail: edgar.andrade@urosario.edu.co

C. Dutilh Novaes

Department of Philosophy - Faculty of Humanities, University of Amsterdam, Oude Turfmarkt 141-147, 1012, Amsterdam, The Netherlands
} 


\section{Introduction}

An important aspect of Brandom's philosophy of language is the reconstruction of "autonomous discursive practices" in terms of propositions, assertions, and declarative sentences $(\mathrm{BSAD}, \mathrm{p} .117) .{ }^{1}$ The latter is a syntactic notion, and we will not deal with it here. The other two notions, namely propositions and assertions, are treated by semantics and pragmatics, respectively.

On the semantic side, the notion of the semantic content of a proposition is defined in terms of its specific inferential articulations (see, for instance, MIE, pp. 133ff). Such inferential articulations are understood in a normative way: "Content is understood in terms of proprieties [in the sense of correctness] of inference, and those are understood in terms of the norm-instituting attitudes of taking or treating moves as appropriate or inappropriate in practice" $(\mathrm{MIE}$, p. 134). Proprieties of inference are normative statuses, and as such they are grounded in the practice of giving and asking for reasons.

According to Brandom, the content of an expression is conferred by the intentional states that ground its use in language. "For it is the practical inferential proprieties acknowledged by such attitudes [of treating an inference as correct or incorrect] that make noises and marks mean what they mean" (MIE, p. 174). Nevertheless, Brandom's explanatory strategy consists in explaining simultaneously both the content of expressions and the content of intentional states. It is a methodological requirement, therefore, that semantics and pragmatics be simultaneously accounted for in terms of the practice of giving and asking for reasons. This leads us to consider this practice in more detail.

In the practice of giving and asking for reasons, the fundamental move is that of making an assertion. To make an assertion is to make a particular move:

According to the model [of the game of giving and asking for reasons], to treat a performance as an assertion is to treat it as the undertaking or acknowledging of a certain kind of commitment ... Doxastic commitments are essentially a kind of deontic status for which the question of entitlement can arise (MIE, p. 142, emphasis in the original).

Being committed and being entitled are normative statuses, which are grounded in the participants' mutual attributions of these statuses to one another. In Brandom's framework, the ability to attribute commitments and entitlements are conditions of possibility for the practice of assertion. Besides this fundamental ability, a story must be told as to how they combine in order to give rise to the inferential practices which eventually constitute the contents of assertions. Indeed, "the inferential articulation [of assertions], in

\footnotetext{
${ }^{1}$ Henceforth, BSAD is an abbreviation for Brandom (2008); MIE is an abbreviation for Brandom (1994); CCDP is an abbreviation for Brandom's unpiblished "Conceptual Content and Discursive Practice".
} 
virtue of which they deserve to be understood as propositionally contentful, consists in consequential relations among the particular doxastic commitments and entitlements" (MIE, p. 142). Thus, not only are the abilities to attribute commitment and entitlement fundamental, but so are the abilities to relate commitments (entitlements) to other commitments (entitlements):

[T] here are two abilities that must be had by any system that can deploy any vocabulary, as part of the autonomous discursive practice of which the use of that vocabulary is a part: the ability to respond differentially to some sentence-tokenings as expressing claims the system is disposed to assert, and the ability to respond differentially to moves relating one set of such sentence-tokenings to another as inferences the system is disposed to endorse ( $\mathrm{BSAD}, \mathrm{p} .44$, emphasis in the original).

Brandom defines three different notions of inferential consequences, based on the notions of commitment and entitlement. They are defined as follows (see BSAD, pp. 120f; MiE, pp. 168-9): ${ }^{2}$

Committive consequence: We say that $p$ committive entails $q$, denoted as $p \square \rightarrow q$, if and only if whenever a speaker $S$ is committed to $p, S$ is committed to $q$.

This kind of inference corresponds to examples like " $A$ is to the West of $B$, so $B$ is to the East of $A$; This monochromatic patch is green, so it is not red" (MIE, p. 168).

Permissive consequence: We say that $p$ permissive entails $q$, denoted as $p \diamond q$, if and only if whenever $S$ is entitled to $p, S$ is prima facie entitled to $q$.

This kind of inference corresponds to examples like "the claim that this is a dry, well-made match can serve as a justification entitling someone to the claim that it will light if struck" (MIE, p. 169). It is worth noting that Brandom's definition of the notion of permissive consequence seems to have changed between MIE and BSAD. This point is relevant for present concerns but we will only come back to it in Section "A Closer Scrutiny of the Hierarchy".

The third inferential relation is based on the notion of incompatibility. We say that $p$ is incompatible with $q$ if and only if commitment to $p$ precludes entitlement to $q$, and commitment to $q$ precludes entitlement to $p$. The notion of incompatibility has been taken to define (part of ${ }^{3}$ ) the semantic content of propositions and performances-the content of $p$ is the class of all $q$ such

\footnotetext{
${ }^{2}$ The symbolic convention is ours. It is also worth noting that, although it is acknowledged that inferences can have more than one premise, for ease of presentation we will restrict ourselves to the single-premise case in this paper.

${ }^{3}$ That the notion of incompatibility only defines part of the semantic content of propositions and performances is relevant for the present purposes. We will come back to this point later on in Section "A Closer Scrutiny of the Hierarchy".
} 
that $p$ and $q$ are incompatible (BSAD, ch. 5) - , and gives rise to the notion of incompatibility entailment:

Incompatibility entailment: We say that $p$ incompatibility entails $q$, denoted as $p \bigcirc q$, if and only if for all $r$, if $r$ is incompatible with $q, r$ is incompatible with $p$.

This kind of inference corresponds to examples like "If my first pet (in fact, let us suppose, a fish) had been a donkey, it would have been a mammal" (BSAD, p. 122).

What is the purpose of these different inferential consequences and what role do they play in Brandom's account? Brandom claims that they are related to-in the sense of 'to represent', or 'to capture' - the familiar inferential relations of deduction and induction. Committive and permissive consequences relate to these familiar notions in the following way: the former corresponds to a generalization to the material case of deductive inferences, and the latter to a generalization to the material case of inductive inferences (BSAD, p. 121; MIE, pp. 168-9). The third inferential relation, incompatibility entailment, is characterized by Brandom as a "counterfactual-supporting, modally robust inferential relation" (BSAD, p. 121). For ease of presentation, we will refer to this relation as robust inference.

Now, the claim that committive and permissive consequences relate to material deductive and inductive inferences is what seems to give substance to the more general and programmatic claim that practical inferential abilities amount to knowledge of the meaning of words and expressions. ${ }^{4}$ Indeed, as we saw above, Brandom contends that meaning can be analyzed in terms of proprieties of inference, which in turn are accounted for in terms of practical inferential abilities. But the claim that meaning can be analyzed in terms of proprieties of inference holds water only if we accept that knowledge of inferential relations can amount to knowledge of meaning. ${ }^{5}$ It is in this sense that it becomes important, for instance, that committive consequence connects with material deductive inferences, so that the committive inference from "A is to the west of $\mathrm{B}$ " to " $\mathrm{B}$ is to the east of $\mathrm{A}$ " can play the role

\footnotetext{
${ }^{4}$ The point is essential in the case of non-logical vocabulary, as opposed to the case of logical constants. In the latter case, both the semantic interpretation of validity and our present-day plurality of logical systems-where the main difference among them lies in their interpretation of logical constants-, makes it natural to connect rules of inference with the meaning of logical constants. The situation is not nearly as natural in the case of non-logical vocabulary. It is the material aspect of inferences that seems to provide the connection with meaning in the case of non-logical vocabulary, and what explains the relevance of connecting committive and permissive consequences with material deductive and inductive inference, respectively.

${ }^{5}$ What Brandom intends is something stronger: that participation in a practice that gives rise to the proprieties of these inferential relations amounts to knowledge of meaning of expressions.
} 
of a 'meaning constitutive' inference of the expressions "west" and "east."6 Thus, the purpose of this connection is to make a discussion of inferential consequences intuitively appealing as an analysis of meaning.

In CCDP (pp. 23-25) Brandom argues that one can use the dimensions of authority and responsibility of assertions in order to determine the following relations between the three inferential consequences:

If $p$ incompatibility entails $q$, then $p$ committive entails $q$.

If $p$ committive entails $q$, then $p$ permissive entails $q$.

In the next section, we show how Brandom constructs this hierarchy of inferential consequences, as discussed in CCDP. In Section "A Closer Scrutiny of the Hierarchy", we present two problems with this hierarchy, one internal and one external. Firstly, by unpacking Brandom's own definitions, we show that a different hierarchy emerges, as both the reciprocal of (1) and the reciprocal of (2) (the latter under certain special conditions) hold. Secondly, with respect to the external problem, we inquire to what extent the hierarchy allows for a connection between Brandom's different notions of inferential consequence and the more familiar notions of robust, deductive, and inductive inferences. We conclude by presenting a number of considerations and open questions raised by our discussion.

\section{The Hierarchy of Inferential Relations}

Let us now look more closely at the way in which Brandom articulates the three inferential consequences in CCDP. The internal role that these inferential consequences play in Brandom's account is to underwrite the inferential practices that eventually constitute the content of assertions. And the way they do this is by playing a particular role in the game of giving and asking for reasons. ${ }^{7}$ The fundamental move in this game is making an assertion, which Brandom accounts for in terms of the notion of "undertaking a commitment" (MiE, p. 167). This is spelled out in terms of the dimensions of authority and responsibility of assertions. Brandom claims that:

In producing assertions, performers are doing two sorts of things. They are first authorizing further assertions (and the commitments they

\footnotetext{
${ }^{6}$ That inductive inferences are also important in their meaning constitutive role depends on Brandom's rejection of a distinction between knowledge of language and knowledge of the world. Such a rejection can be best appreciated in his discussion of rational rectification (cf. BSAD, ch. 6), but a discussion of this aspect of Brandom's philosophy of language is beyond the scope of this paper.

${ }^{7}$ For reasons of space we cannot give a detailed account of this role, but see MIE, pp. $190 \mathrm{ff}$.
} 
express), both concommitant commitments on their part (inferential consequences) and claims on the part of their audience (communicational consequences). In doing so, they become responsible in the sense of answerable for their claims. That is, they are also undertaking a specific task responsibility, namely the responsibility to show that they are entitled to the commitment expressed by their assertions, should that entitlement be brought into question (MIE, p. 173, emphasis in the original).

The gist of the argument in the construction of the hierarchy based on (1) and (2) is to take the dimensions of authority and responsibility as a characterization of doxastic commitments. It is in virtue of this characterization that it can be proved that a scorekeeper is committed to a claim, which is a necessary step in the proof of (1), as we shall see in a moment. To this effect, suppose that $S$ is a speaker and $p$ a sentence. What $S$ does when she asserts $p$ is to undertake (and acknowledge) a commitment, which can be spelled out along the two dimensions of responsibility and authority as follows. We say that $S$ is committed to $p$ if and only if:

Responsibility: $S$ has to respond to any challenge to $p$.

Authority: $S$ authorizes others to be committed to $p$. This means that $S$ has to be prepared to make his own any challenge to $p$ addressed to others. Or, conversely, that others can discharge responsibility to show entitlement to $p$ by deferring it to $S$.

Besides the two dimensions of responsibility and authority, an analysis of commitment requires us to spell out in somewhat more detail the notion of challenge. In the model of giving and asking for reasons, challenge is an important notion; it is a speech-act that constitutes a move in the game of giving and asking for reasons. However, in line with Brandom's contention that assertion is the fundamental speech-act (MIE, p. 172), a challenge is understood in terms of assertion (otherwise assertion would not be the basic speech-act Brandom deems it to be). Therefore, one "can challenge an assertion only by making an assertion incompatible with it" (MIE, p. 178).

With these definitions at hand, we first prove that $p \circ \rightarrow q$ entails $p \square \rightarrow q$, that is, (1), and next we proceed to proving (2).

Proof In order to prove (1) suppose that $p \bigcirc \rightarrow q$ and that $S$ is committed to $p$. We show that $S$ is committed to $q$ by showing (i) that $S$ has to respond to any challenge to $q$; and (ii) that $S$ authorizes others to assert $q$ :

(i) Suppose $q$ is appropriately challenged with $r$, which is incompatible with $q$. Then $p$ is appropriately challenged with $r$ as well, because $p \circ \rightarrow q$ and therefore $r$ is incompatible with $p$. In other words, if $p \rightarrow \rightarrow q$ and $r$ is a challenge to $q$, then $r$ is a challenge to $p$. Furthermore, since $S$ is committed to $p$, he has to respond to $r$. Thus, we have just shown that $S$ has to respond to any challenge to $q$.

(ii) By assumption, $S$ authorizes others to be committed to $p$. This means that he will make his own any challenge to $p$ addressed to other people. But 
since any challenge to $q$ is a challenge to $p$, he also has to make his own any challenge to $q$.

This proves that $p \bigcirc \rightarrow q$ entails $p \square \rightarrow q$. Now we turn to the proof of (2):

Proof Suppose that $p \square \rightarrow q$ and that $S$ is entitled to $p$. In his presentation of the game of giving and asking for reasons, Brandom states that $S$ "can [be] attribute[d] entitlement to any claims that are committive-inferential consequences of commitments to which [she] is already taken to be entitled" (MIE, p. 191). This means that, given $p \square \rightarrow q$, unless $S$ is entitled to $q$, he cannot be entitled to $p$. But since we have assumed the latter, $S$ has to be entitled to $q .{ }^{8}$

Brandom claims that the order of this hierarchy is "strict"-i.e., that the 'if... then' in (1) and (2) are not reversible, although he does not give any explicit reason for it. One argument that might support the claim that (2) is not reversible is the following construction of a counterexample (which, by the way, we will challenge below). Consider how Brandom defines permissive consequence as inheritance of entitlement in MIE:

Inheritance of entitlement (being entitled to one claim as a consequence of entitlement to another) is what will be called a permissive, or entitlement-preserving inferential relation. Inductive empirical inferences exploit relations of this genus. The premises of these inferences entitle one to commitment to their conclusions (in the absence of countervailing evidence) but do not compel such commitment. For the possibility of entitlement to commitments incompatible with the conclusion is left open (MIE, p. 168, emphasis in the original).

This definition leaves room for the following interpretation. Permissive inference comes down to inheritance of entitlement. If $p \diamond \neg q$ and $S$ is entitled to $p, S$ is entitled to $q$. The claim that a permissive inference is defeasible consists in the following. While $S$ is entitled to $q$, it could be the case that he cannot commit to it despite his commitment to $p$. For $S$ could already be entitled to $r$, where $r$ is incompatible with $q$, but not with $p$. Thus, should $S$

\footnotetext{
${ }^{8}$ More than a proof, this is just one way to formulate the articulations involved in the game of giving and asking for reasons. In fact, (2) is explicitly stated in a discussion of such articulations: "If one is entitled to $p$ and $p$ commitment-entails $q$, one is entitled to $q$-any entitlement-defeating incompatibilities to $q$ equally defeat entitlement to $p$ " (MIE, endnote 43, p. 674). The proof that appears in CCDP is the following: "We can see in much the same way why if there is a commitment-preserving inference from $\mathrm{p}$ to $\mathrm{q}$, there is also a (prima facie) entitlement-preserving inference from $\mathrm{p}$ to $\mathrm{q}$. For if everyone who is committed to $\mathrm{p}$ is thereby committed to $\mathrm{q}$, then on the side of authority, in authorizing others to assert $\mathrm{p}$, I am thereby authorizing them to assert q. And to say that is to say that they can inherit entitlement to q from my entitlement to p. And on the side of responsibility, in undertaking the responsibility to justify or otherwise vindicate my entitlement to $\mathrm{p}$, I am thereby undertaking the responsibility to justify, or otherwise vindicate my entitlement to q. So I cannot be entitled to p unless I am also entitled to q, which is to say that there is a good inference from $p$ to q preserving prima facie entitlements" (CCDP, p. 24).
} 
commit to $p$, this will not be followed by a commitment to $q$. Clearly, this implies that $p \square \rightarrow q$ is not valid. ${ }^{9}$

\section{A Closer Scrutiny of the Hierarchy}

Brandom claims (CCDP, p. 25) that the 'if... then' in (1) and (2) are not reversible. However, under a closer scrutiny, there are reasons to doubt the accuracy of this claim. Indeed, we shall prove, on the basis of Brandom's own definitions, that the converse of (1) holds. In other words, we can prove that (3) holds:

If $p$ committive entails $q$, then $p$ incompatibility entails $q$.

This means that if (1) holds, Brandom's own conceptualization of the notions of incompatibility entailment and committive consequence allows the two notions to collapse into each other: a claim not without consequences, as we will show below. The proof of (3) is the following:

Proof Suppose that $p \square \rightarrow q$. We need to show that $p \bigcirc \rightarrow$. Let $r$ be such that it is incompatible with $q$. We shall prove that $r$ is incompatible with $p$. Consider the notion of $p$ being incompatible with $r$ paraphrased as follows: ${ }^{10}$

(i) If $S$ is committed to $p, S$ is not entitled to $r$.

(ii) If $S$ is committed to $r, S$ is not entitled to $p$.

(i) If, on the one hand, we assume that $S$ is committed to $p$, by the assumption that $p \square \rightarrow q$ it follows that $S$ is committed to $q$. Since $r$ is incompatible with $q, S$ cannot be entitled to $r$.

(ii) On the other hand, if we assume that $S$ is committed to $r$, since $r$ is incompatible with $q$, then $S$ cannot be entitled to $q$. This means that if $S$ were to assert $q$, he could not fulfill his responsibility to show entitlement to $q$. This would not be the case if $S$ were entitled to $p$, since he could discharge his responsibility with respect to $q$ by asserting $p$, given that $p \square \rightarrow q \cdot{ }^{11}$ Therefore, $S$ is not entitled to $p$.

We have just shown that $r$ is incompatible with $p$. Therefore, if $p \square \rightarrow q$, then $p \bigcirc \rightarrow$. In view of (1), we have that $p \square \rightarrow q$ if and only if $p \bigcirc \rightarrow$.

Moreover, under certain conditions, we can prove (4):

If $p$ permissive entails $q$, then $p$ incompatibility entails $q$.

\footnotetext{
${ }^{9}$ It is worth noting that this version of defeasibility is not in conflict with the assumption that the counterpositive of a permissive inference is valid.

${ }^{10}$ It might be worth stressing that this paraphrase is nothing over and above Brandom's own definition of incompatibility. It is just a slightly different way of presenting the original definition. 11 "If one is entitled to $p$ and $p$ commitment-entails $q$, one is entitled to $q$ " (MIE, endnote 43, p. 674).
} 
The first condition states that the incompatibility relation is symmetrical, in the sense that if one denies that $p$ and $q$ are incompatible, one is denying both (i) If $S$ is committed to $p, S$ is not entitled to $q$, and (ii) If $S$ is committed to $q, S$ is not entitled to $p$. In other words, we must assume that (i) if and only if (ii). We take it that this is what Brandom has in mind when he imposes the condition of symmetry over the incompatibility relations that are "suitable as semantic primitives" (BSAD, p. 123). ${ }^{12}$

The second condition is contentious. We must assume that the counterpositive of a permissive consequence is valid. That is, from the fact that $S$ is not entitled to $q$ we should be able to derive that $S$ is not entitled to $p$. This assumption is contentions because permissive consequence is meant to be defeasible (or valid only prima facie). ${ }^{13}$

Under these conditions we can prove (4):

Proof We prove that if $p \diamond \rightarrow q$ then $p \circ \rightarrow q$. Suppose that $p \diamond \rightarrow q$ and that $r$ is incompatible with $q$. The latter assumption implies that if $S$ is committed to $r$, $S$ cannot be entitled to $q$. Since entitlement to $p$ implies entitlement to $q$, by contraposition it follows that $S$ cannot be entitled to $p$ either. By symmetry of incompatibility, it follows that $r$ is incompatible with $p$.

We can use (4) to show that the argument used at the end of Section "The Hierarchy of Inferential Relations" to produce a counterexample of the reverse of (2), that is, that permissive consequence entails committive consequence, is no longer sound. Under the assumptions that $p \diamond \rightarrow q$ and that $S$ is entitled to $p$, the argument is intended to show that $p \square \rightarrow q$ is not valid, that is, that $S$ can be committed to $p$ without being committed to $q$. The argument requires one to assume that $S$ is already entitled to $r$, where $r$ is incompatible with $q$, so that $S$ cannot be committed to $q$. But the argument also requires that it be possible for $r$ to be incompatible with $q$ without being incompatible with $p$. However, by (4), $p \diamond q$ entails $p \bigcirc \rightarrow$. Thus, $r$ is also incompatible with $p$. Therefore, $S$ cannot be committed to $p$ and thus we cannot show that $p \square \rightarrow q$ is not valid.

With this counterexample out of the way, we can prove that the reciprocal of (2), that is (5), holds (under the condition that (4) holds):

If $p$ permissive entails $q$, then $p$ committive entails $q$.

Proof The proof of (5) follows straightforwardly from (4) and (1).

\footnotetext{
12 "I will only consider symmetric incompatibility relations. This is an intuitive condition because it is satisfied by familiar families of incompatible properties: colors, shapes, quantities, biological classifications, and so on" (BSAD, p. 123).

${ }^{13}$ Robert Brandom pointed out this caveat during the presentation of this paper at the Workshop on Brandom's Analytic Pragmatism in Genoa, April 2009. Pete Wolfendale made a similar point in personal communication.
} 
If the proof of (4) is indeed sound, not only is permissive consequence equivalent to committive consequence (in virtue of (2) and (5)), but all three consequences, on Brandom's own definitions, turn out to be equivalent. Note that we are not suggesting that there is no genuine distinction between these three notions. Rather, what we suggest is that Brandom's own formulation of them does not succeed in capturing the distinction, if indeed there is one.

It turns out that Brandom adapted, or rather, clarified, his definition of permissive consequence in $\mathrm{BSAD}$ :

And one takes or treats $q$ as an inferential consequence of $p$ in another sense by being disposed to attribute entitlement to the claim that $q$ to whomever one credits with entitlement to the claim that $p$. As will appear, entitlement-preserving inferences are always defeasible; the entitlement one acquires thereby is only prima facie. One is not entitled to the conclusion of a good entitlement-preserving inference if one is committed to something incompatible with it. (BSAD, pp. 119f including footnote 2.)

In this case, the counterpositive of a permissive consequence is not valid, that is, one can be entitled to the antecedent and yet find out that one cannot be entitled to the consequent. Thus, the proof of (4) does not go through. What remains unclear, however, is the fact that, given (2), there are permissive consequences the counterpositives of which are valid, namely, the permissive consequences that we can obtain from committive consequences following Brandom's own endorsement of (2). The latter are not defeasible, ${ }^{14}$ so they give rise to undefeasible permissive consequences. This seems problematic given Brandom's own claim that "[a]s will appear, entitlement-preserving inferences are always defeasible" (BSAD, p. 120, emphasis added).

Nevertheless, to bring the internal criticism of the hierarchy to a close, we claim that the equivalence between incompatibility entailment and committive consequence holds, while the equivalence between committive and permissive consequences is more problematic. In any case, this shows that, on the basis of Brandom's own arguments and definitions, the hierarchy is different from what he claimed it to be in CCDP.

Now, turning to the external criticism of the hierarchy, a few points call for a closer inspection, given that the connections among the three inferential consequences have repercussions for the possibility of relating them to more familiar notions of inferential relations. We have seen in Section "Introduction" that such a connection is paramount for Brandom's overall project of analyzing meaning in terms of inferential practices.

The first difficulty is that (2), which connects committive consequence with permissive consequence, is somewhat odd, in that it implies that any deductive

\footnotetext{
${ }^{14}$ That committive consequences are not defeasible can be attested by the following claim: "The first sort, commitment-preserving inferential relations, is a generalization, to include the case of non-logical, material inferences, of obligatory, deductive inferential relations" (BSAD, p. 120, emphasis added).
} 
inference corresponds to an inductive inference. In other words, since $p \square \rightarrow q$ means that there is a sound (material) deduction from $p$ to $q$, (2) implies that there is a plausible (material) induction from $p$ to $q$. This does not make much sense. Deduction and induction are categorically different concepts, not concepts that differ in 'strength' ${ }^{15}$ Such a distinction points towards what may seem the right interpretation of the proof of (2). For this proof shows that from $p \square \rightarrow q$ we can deduce that entitlement to $q$ is inherited from entitlement to $p$. However, the definition of permissive consequence is that from entitlement to $p$ we can 'defeasibly infer' entitlement to $q$, that is, permissive consequence does not refer to inheritance of entitlement. In other words, the proof of (2) does not connect committive consequence and permissive consequence (despite Brandom's own claim in CCDP that it does).

Now, we must bring to the fore the fact that an adequate semantics for natural language cannot be constructed only on the basis of the notion of incompatibility. Brandom is clear in this respect: "Here is a semantic suggestion: represent the propositional content expressed by a sentence with the set of sentences that express propositions incompatible with it. [fn. 5: Since, as has just been emphasized, incompatibility relations are only one dimension of inferential articulation, this semantic representation of conceptual content will necessarily be only partial.]" (BSAD, p. 123). But why are the other dimensions of inferential articulation important? Brandom claims that "any autonomous discursive practice (ADP) must include practices-or-abilities of distinguishing some inferences as materially good from others that are not. For some bit of vocabulary to function as a propositionally contentful declarative sentence is for it to be available to serve as the premise and conclusion of such material inferences" (BSAD, pp.103f). One reason Brandom relies on a three-fold inferential articulation, which includes committive and permissive consequences besides incompatibility entailments, is that incompatibility entailment is monotonic, ${ }^{16}$ whereas material inferences are non-monotonic (BSAD, pp. 106f). A complete account of (meaning-constitutive) material

\footnotetext{
${ }^{15}$ That this is so can be attested by the monotonicity of deduction, as opposed to the nonmonotonicity of induction. The monotonicity of the former, and the non-monotonicity of the latter, make part of the informal notions of deduction and induction. We acknowledge the fact that part of Brandom's program is precisely to give a different analysis of these terms, for instance, by taking a primitive notion of materially valid inferences that is prior to a discussion of preservation of truth. But of course, his analysis is still accountable towards the well-established, informal notions of deduction and induction. Brandom also seems to assume that this distinction in terms of (non-)monotonicity exists in the case of deduction and induction (cf. BSAD, p. 120).

${ }^{16}$ In fact, it can be proved that incompatibility entailment is monotonic, given that the relation of incompatibility is governed by the principle of persistence. Persistence of incompatibility says that "if one set of claims is incompatible with another, so too is any larger set containing it. That is, one cannot remove or repair an incompatibility by throwing in some further claims" (BSAD, p. 123). In particular, the principle of persistence says that if $p$ and $q$ are incompatible, so are $\{p\} \cup \Gamma$ and $q$. This principle entails monotonicity of incompatibility entailment. Suppose $p$ incompatibility entails $q$. Does $\{p\} \cup \Gamma$ incompatibility entail $q$ ? The answer is: yes. If $s$ is incompatible with $q$, it is incompatible with $p$, and hence it is incompatible with $\{p\} \cup \Gamma$.
} 
inferences requires a notion of non-monotonic inference that incompatibility entailment cannot provide, or at least not on its own. ${ }^{17}$ Note that the monotonicity of incompatibility entailment, and the non-monotonicity of permissive consequence, is yet another reason, in view of (1), to reject (2). ${ }^{18}$

Moreover, as a second difficulty, Brandom claims that committive consequence is intended to correspond to deductive (material) inference and that incompatibility entailment is intended to correspond to robust inference. The question now is whether, given their equivalence, we can keep the promise of this correspondence. In the end, the gist of the problem is whether the notion of deductive consequence is the same as, or equivalent to, the notion of robust inference. According to Brandom, the notion of incompatibility entailment has an intrinsic modal ingredient. He presents this in terms of an example:

The fact that the properties of being a donkey and being a mammal stand in the relation of incompatibility entailment means that every property incompatible with being a mammal is incompatible with being a donkey. ... We could say: "Necessarily anything that is a donkey is a mammal" (BSAD, pp. 122, emphasis in the original).

However, compare this quote with a traditional formulation of deductive inference, namely the one offered by Aristotle in the Prior Analytics. Clearly, it also has a modal component from the start:

A deduction is a discourse in which, certain things being stated, something other than what is stated follows of necessity from their being so (Aristotle, Prior Analytics 24b19-20, emphasis added).

There is nothing distinctive in Brandom's presentation of robust inference in terms of incompatibility entailment that is not already captured by the traditional notion of deductive inference. We take it that a consequence of (1) and (3) is that, thus formulated, the notion of robust consequence collapses into the notion of deductive inference. ${ }^{19}$

It is worth noting that, if our intuitions about robust inference were stronger-that is, sharply distinct from our intuitions about deductive inference-, on the face of (1) and (3) we would have to revise the definition

\footnotetext{
${ }^{17}$ The step from the more familiar idea that (non-monotonic) material inferences bestow meaning on sentences-e.g., that it is in virtue of the meanings of "west" and "east" that there is a material inference from " $\mathrm{A}$ is to the west of $\mathrm{B}$ " to "B is to the east of $\mathrm{A}$ "-, to the claim that "there is an intimate connection between the conceptual contents expressed by vocabularies and the counterfactually robust inferences they are involved in" (BSAD, p. 122), depends on an argument about 'tractability' of the "practical task of updating the rest of one's beliefs when some of them change" (BSAD, p. 109) the discussion of which is beyond the scope of this paper.

${ }^{18}$ The gist of the points in this paragraph has been suggested by an anonymous referee.

${ }^{19}$ While there does not seem to be a difference between robust and deductive consequence, there is, however, an important difference between incompatibility entailment and committive consequence: the fact that incompatibility entailment is essentially an ontic notion while committive entailment is essentially a pragmatic notion (cf. BSAD, pp. 191ff). But this is not the place to pursue this criticism.
} 
of either incompatibility entailment or of committive consequence in order to justify the claim that they are distinct and thus to save the intended connections between Brandom's notions and the more 'familiar' ones. Alternatively, (1) and (3) may in fact suggest the need for a revision of our own intuitions about robust inference.

\section{Conclusion}

In this paper we have explored the internal logical relations between the definitions offered by Brandom himself of three kinds of entailment relations: incompatibility entailment, committive consequence and permissive consequence. We conclude that the idea that incompatibility entailment, committive consequence, and permissive consequence correspond to robust inference, deductive inference, and inductive inference, respectively, must be abandonedat least if robust inference is meant to be something different from deductive inference. There are no three different notions of inference emerging from Brandom's definitions, but at most two. And insofar as Brandom's inferentialist pragmatism relies crucially on the identification of robust inference with incompatibility entailment, of deductive inference with committive consequence, and of inductive inference with permissive consequence, the results presented here may have important implications also for the cogency and strength of his general program. But for now this will remain a topic for further research.

Acknowledgements We would like to thank Martin Stokhof, Pete Wolfendale and an anonymous reviewer for their insightful comments on earlier drafts. We would also like to thank Robert Brandom and the participants of the workshop "Towards an Analytic Pragmatism" held in Genoa, 2009 , for their comments. This research was conducted while the first author was working at the Institute for Logic, Language, and Computation, and he is grateful for the support that he received from it during this time.

Open Access This article is distributed under the terms of the Creative Commons Attribution Noncommercial License which permits any noncommercial use, distribution, and reproduction in any medium, provided the original author(s) and source are credited.

\section{References}

Brandom, R. B. (1994). Making it explicit: Reasoning, representing, and discursive commitment. Harvard University Press.

Brandom, R. B. (2008). Between saying and doing: Towards an analytic pragmatism. Oxford University Press. 